\title{
Short Fiber Reinforced Thermoplastics: Prediction of Stiffness in Injection Molded PS-PPO Blends
}

\author{
Bora TeKKanaT* AND Ronald Gibala \\ Materials Science and Engineering Department \\ University of Michigan \\ Ann Arbor, MI 48109, USA
}

\begin{abstract}
The prediction of stiffness in short fiber reinforced thermoplastics is studied as a function of fiber length using injection molded blends of PS and PPO. The theoretical models for predicting composite stiffness are reviewed. The results are first compared with the theoretical models advanced for uniaxially aligned composites. These models predict higher than experimental values. However, agreement between the predictions and experimental values improves when the effect of fiber orientation distribution in the injection molded samples is taken into account and as the ductility (or the PPO content) of the matrix increases. Cox's model when used with the "laminate analogy" gives the closest prediction to the experimental stiffness. Reinforcement efficiency factor for stiffness is a strong function of retained fiber lengths. The dependence of composite stiffness on the matrix ductility and the effects of compatibility on the mechanical properties of PS-PPO blend system are also discussed.
\end{abstract}

\section{INTRODUCTION}

QHORT FIBER REINFORCED thermoplastics (SFRTP) are commercially very atStractive. They can be processed using rapid fabrication techniques, e.g., injection molding while retaining many benefits of more conventional composites. These materials are already being used successfully in the automotive industry, consumer appliances, commercial business machines and in several other applications. Prediction of the performance of injection molded SFRTP is desirable from the design standpoint. However, the prediction is difficult, since the mechanical properties are complicated by the nonuniformity of the fiber length, and the distribution of the fiber orientation. One of our aims in this study is to explore the predicted dependence of composite stiffness as a function of fiber length for the SFRTP.

\footnotetext{
*To whom correspondence should be addressed. Present address: Johnson Controls, Inc., Milwaukee, WI 53201, U.S.A.
} 
The polystyrene (PS)-polyphenylene oxide (PPO) blend system is studied for representing a wide range of matrix materials. The blends of PS and PPO show a complete solid solubility on the molecular level over the entire range of compositions [1-5]. More important, the PS-PPO blend system shows a transition from brittle to ductile failure with increasing PPO content by a gradual change in the microdeformation mechanisms $[4,6]$. The matrix ductility can, therefore, be easily controlled with the composition. Furthermore, both PS and PPO are amorphous polymers, hence the problems associated for a semicrystalline matrix are avoided. In a semicrystalline matrix, the morphology and properties become more sensitive to the processing conditions and to the presence of fibers.

A study of the literature showed that little work has been reported that deals with the predictions of stiffness versus fiber length in well characterized SFRTP. The most convenient composites to study in a predictive work are those having a simple arrangement of fibers, e.g., fully aligned or random in a plane for which the theoretical predictions have been advanced. It is, however, difficult in practice to make such model composites. In this study, injection molding was used to prepare the samples. Although very complex parts can be produced at very high production rates with this technique, it does not permit the control of fiber orientation in the part and significantly limits the fiber length that can be retained. A molded component usually shows a very complex distribution of fiber orientations which can vary from one point to another. We focused our attention to the simplest theories for the prediction of composite stiffness in SFRTP which would be easy to use and would be accurate over a wide range of materials.

\section{THEORY}

For continuous and unidirectional fiber composites, stiffness is predicted by the "Rule of Mixtures" (ROM). In this model, the modulus of composite $E_{c}$ is given by:

$$
E_{c}=E_{f} v_{f}+E_{m}\left(1-v_{f}\right)
$$

$E_{m}$ and $E_{f}$ are the elastic moduli of the matrix and the fiber, respectively, and $v_{f}$ is the fiber volume fraction.

\subsection{Stiffness of Short Fiber Composites}

Equation (1) may be modified by including a length correction factor, $\eta_{\ell}$, for uniaxially aligned fibers of finite length.

$$
E_{c}=E_{f} v_{f} \eta_{\ell}+E_{m}\left(1-v_{f}\right)
$$

This length correction factor is given by $\operatorname{Cox}[7]$ as:

$$
\eta_{\ell}=1-\left[\frac{\tanh (\beta \ell / 2)}{(\beta \ell / 2)}\right]
$$


$\ell$ is the fiber length and $\beta$ is given by:

$$
\beta=\left[\frac{2 \pi G_{m}}{E_{f} A_{f} \ln (R / r)}\right]^{1 / 2}
$$

In Equation (4), $A_{f}$ is the cross-sectional area of the fiber, $G_{m}$ is the shear modulus of matrix, $r$ is the fiber radius, and $R$ is the average distance between fibers normal to their length. The size of $\beta$ will determine the dependence of $E_{c}$ on fiber length. To get the length correction factor, Cox assumed the fibers had hexagonal arrangement and this distribution was uniform throughout the cross section of the composite. The value of $\beta$ can be evaluated from the above equation given the values for $G_{m}, E_{f}, A_{f}$, and $v_{f}$. It is shown by the following relation that, for hexagonal distribution of fibers, volume fraction of fibers can be calculated in terms of $r$ and $R$.

$$
v_{f}=\left[\frac{2 \pi r^{2}}{\sqrt{3} R^{2}}\right]
$$

Certain assumptions were made in Cox's analysis and it is important to point them out here. First, the matrix and fibers were assumed to be strained elastically, and second, there was no relative movement at the fiber-matrix interface under applied loads, i.e., the displacements of the fiber, matrix, and composite were the same during loading $\left(e_{f}=e_{m}=e_{c}\right)$. Lastly, the fibers were assumed to be parallel and aligned unidirectionally in the intended loading direction.

Halpin and Tsai [8] also developed a simple generalized stiffness equation reduced from Hermans' solution [9] to Hill's "self-consistent method" [10]:

$$
E_{\mathrm{c}}=E_{m}\left[\frac{\left(1+2 A v_{f} \eta\right)}{\left(1-v_{f} \eta\right)}\right]
$$

$A$ is equal to $\ell / d, d$ is fiber diameter, and

$$
\eta=\left[\frac{\left(E_{f} / E_{m}\right)-1}{\left(E_{f} / E_{m}\right)+2 A}\right]
$$

In Equation (6) the composite stiffness depends on the fiber aspect ratio.

The last model to be considered for the stiffness prediction in this study is the Ogorkiewicz and Weidmann [11] equation which is based on the model by Counto [12]. Ogorkiewicz and Weidmann assumed a "prism-within-prism" distribution for a rectangular prism of the fiber material, with its long axis parallel to the direction of fiber alignment and the applied tensile load, within a prism of the plastic matrix. The equation is based on the other assumption that there is a perfect bonding between the fiber and matrix:

$$
E_{c}=E_{m}\left[\frac{E_{f}\left(A v_{f}+\sqrt{v_{f}}-v_{f}\right)+E_{m}\left(A-A v_{f}-\sqrt{v_{f}}+v_{f}\right)}{E_{f}\left(\sqrt{v_{f}}-v_{f}\right)+E_{m}\left(A-\sqrt{v_{f}}+v_{f}\right)}\right]
$$


The list of works presented above is not exhaustive. These models were considered here because they are simple and they represent different approaches to the problem.

\subsection{Stiffness of Partially Oriented Composites}

The discussion so far has been concerned with the stiffness of uniaxially aligned composites. However, the production techniques usually used with SFRTP do not permit control of fiber orientation. Any predictive work will be of little practical value unless the fiber orientation distribution (FOD) is taken into account in theoretical analyses. Krenchel [13] developed an "orientation efficiency factor," $\eta_{0}$ to account for the anisotropy of stiffness in Equation (2).

$$
E_{c}=E_{f} v_{f} \eta_{\ell} \eta_{o}+E_{m}\left(1-v_{f}\right)
$$

The orientation efficiency factor $\eta_{o}$ is determined by dividing the reinforcement into groups of uniaxially aligned fibers:

$$
\eta_{o}=\sum_{h} a_{h} \cos ^{4} \theta_{h}
$$

where

$$
\sum_{h} a_{h} \doteq 1
$$

$a_{h}$ is the fiber fraction oriented at an angle $\theta_{h}$ to the applied load. Equation (9) represents a simple method for the prediction of the stiffness of SFRTP. However, this equation predicts that fibers oriented perpendicular to the applied load offer no contribution to the matrix stiffness.

An alternate treatment of the stiffness of a composite in which there is a complex fiber orientation distribution and fiber length distribution was developed by Halpin and Pagano [14]. The approach adopted which is known as the classical "laminated plate theory" (LPT) or "laminate analogy" (LA), treats the composite as an assembly of laminates. The theory proposes that the stiffness of each layer is predicted or measured experimentally and the stiffness of the "plies" are summed to give the stiffness of the composite. The thickness of a ply $(\Delta h)$ unidirectionally aligned at an angle $(\theta)$ to the reference axis in the laminated composite is considered proportional to the fiber fraction $\left(a_{h}\right)$ having an angle $(\theta)$ in the fiber orientation distribution of the short fiber composite.

$$
E_{\mathrm{c}}=\sum \frac{\Delta h}{h} E_{h}
$$

where

$$
h=\sum_{h} \Delta h
$$


Although there may appear to be little difference between the Krenchel and Halpin methods as far as the form of the final equations are concerned, in the former a general efficiency factor is being evaluated whereas the latter sums the stiffness of individual fractions of fibers aligned at an angle $\theta$. The laminate analogy opens many possibilities for micromechanics to be used in the prediction of stiffness of an individual ply containing uniaxially aligned discontinuous fibers. The success of the lamination approximation is strongly dependent upon the physical volume averaging in real material systems combined with an ability to estimate the stiffness for an oriented short fiber sheet as a function of constituent properties, concentrations, and aspect ratios.

\section{EXPERIMENTAL}

The materials used in this study are a general purpose atactic polystyrene (PS) from Dow Chemical, a poly-2,6-dimethyl-1,4-phenylene oxide (PPO) from General Electric, and $1 / 4,1 / 8$, and $1 / 16$ " long chopped fiberglass from OwensCorning. Glass fibers supplied had diameters between 8.89 and $10.15 \mu \mathrm{m}$ and an organosilane coupling agent on them. Molecular weight of polymers was measured with a Water's 150-C gel permeation chromatography (GPC) system. PS has a $M_{W}=300,000$ and $M_{N}=150,000$ and PPO has a $M_{W}=50,000$ and $M_{N}=20,000$. Properties of the material used are given in Table 1 .

Blends of PS and PPO were prepared by mechanical mixing in a $3 / 4$ " single screw extruder. Homopolymers were dry mixed by weight for each specific composition $(25,50,65,75$, and $85 \%$ PPO by weight) and extruded twice to ensure a proper blending. The glass-transition temperatures $\left(T_{g}\right)$ of PS, PPO, and their blends were determined by differential scanning calorimetry (DSC) with a Perkin-Elmer DSC-II. 10, 20, and 30 weight \% glass filled compositions of polymers with three fiberglass types $\left(1 / 4,1 / 8\right.$, and $1 / 16^{\prime \prime}$ chopped fibers) were prepared by melt coupling compounding in the same extruder used for blending.

ASTM "D638 Type-V" tensile bars were injection molded for each composition into a single cavity mold with an end gate by using a 45 ton Battenfeld injection molding machine. The mechanical properties were determined on the Instron tensile machine at a strain rate of $6.6 \times 10^{-4} / \mathrm{sec}$ in air at room temperature.

Table 1. Properties of the material used.

\begin{tabular}{lccc}
\hline Property & PS & PPO & Fiberglass \\
\hline $\begin{array}{l}\text { Specific gravity } \\
\left(\mathrm{g} / \mathrm{cm}^{3}\right)\end{array}$ & 1.05 & 1.07 & 2.54 \\
$\begin{array}{l}\% \text { Elongation } \\
\text { at break }\end{array}$ & 5.4 & $>20.0$ & 4.8 \\
$\begin{array}{l}\text { Elastic modulus } \\
\quad(\mathrm{GPa})\end{array}$ & 2.37 & 1.65 & 72.40 \\
$\begin{array}{l}\text { Tensile strength } \\
(\mathrm{MPa})\end{array}$ & 49.6 & 68.9 & 3448 \\
\hline
\end{tabular}


Table 2. Properties of the PS/PPO blend system.

\begin{tabular}{lcccc}
\hline $\begin{array}{l}\text { Composition } \\
\text { (weight \%) }\end{array}$ & $\begin{array}{c}E \\
\text { (GPa) }\end{array}$ & $\begin{array}{c}S \\
(\mathrm{MPa})\end{array}$ & $\begin{array}{c}\text { \% Elong. } \\
\text { at Break }\end{array}$ & $\begin{array}{c}\boldsymbol{T}_{\boldsymbol{g}} \\
\left({ }^{\circ} \mathrm{C}\right)\end{array}$ \\
\hline PS & 2.37 & 49.6 & 5.4 & 110 \\
75PS-25PPO & 2.42 & 67.8 & 6.4 & 122 \\
50PS-50PPO & 2.09 & 70.6 & 7.0 & 146 \\
35PS-65PPO & 1.93 & 73.9 & 7.4 & 159 \\
25PS-75PPO & 1.82 & 74.8 & 8.6 & 173 \\
15PS-85PPO & 1.76 & 76.9 & $>16.0$ & 190 \\
PPO & 1.65 & 68.9 & $>20.0$ & 210 \\
\hline
\end{tabular}

To get detailed information on fiber length distribution (FLD) of a molded part, considerable experimental care must be exercised. Although it is a tedious procedure, FLD is best gotten by manual measurement of the lengths of many fibers taken from a representative sample. The fibers were first removed from the matrix by chemical digestion using solvent, then recovered on a glass slide and photographed under a microscope for length measurement, at least 1000 fibers were measured for each composition characterized. Actual fiberglass contents were measured by pyrolysis.

FOD was determined by taking thin sections parallel to the flow direction from the gauge area of the tensile test specimens and using transmission light microscopy. Metallographic technique described by Holik et al. [15] was followed for the preparation of $25-50 \mu \mathrm{m}$ thick sections used in FOD determinations. Since injection molded samples have a complex fiber orientation distribution through the sample thickness, three sections were taken for each sample and the distributions of each section were summed to determine the resulting FOD.

\section{RESULTS AND DISCUSSION}

\subsection{Mechanical Behavior of the PS-PPO Blend System}

The blends of PS and PPO exhibited a single $T_{g}$ between the $T_{g}$ 's of the two components. This points out a complete compatibility between the homopolymers. The strong interaction, determined using FTIR, between the phenyl ring of PS and the phenylene ring of PPO was thought by Wellinghoff and Baer [3] to be responsible for the blend compatibility.

Characterization of the mechanical behavior of the PS-PPO blend system included the measurement of elastic modulus, tensile strength, percent elongation at break, and mode of failure. The discussion here is intended to provide some understanding for the observed behavior of this system and the effects of compatibility on the mechanical properties of PS-PPO blend system. A summary of the mechanical properties, and $T_{g}$ 's for the system are given in Table 2 . Both elastic modulus and tensile strength for the blends are higher than the linear combination of the values for the pure components. The similar behavior was also noted 
by Yee and Maxwell [5] in compression. They also observed a negative excess volume of mixing because of blending. They reasoned this to be responsible for a stiffening of the solid lattice and the reduction in chain mobility to explain the observed elastic modulus and strength behavior of the PS-PPO blend system. The values show a maximum around $25 \%$ PPO for elastic modulus and $85 \%$ PPO for tensile strength. Results of \% elongation at break show a gradual increase in ductility as the PPO content increases. However, it is difficult to determine the mode of failure solely from the stress-strain behavior. Failure mechanisms of the PSPPO blends are studied separately [16] in detail using subsurface analysis by transmission optical microscopy and fracture surface analysis by scanning electron microscopy (SEM). In that study, it was established that the compositions with $0-50 \%$ PPO content failed brittlely while the compositions with $65 \%$ and higher PPO content showed a ductile failure.

\subsection{Reinforcement Efficiency}

The reinforcement efficiency is viewed here for stiffness. The stiffness of short fiber reinforced thermoplastics depends on the fiber length and its distribution, volume fraction of fibers, the stress transfer efficiency of the interface and the fiber orientation distribution.

The most direct verification of reinforcement efficiency can be done by comparing the experimental values of well-defined fiber lengths with those predicted by the rule of mixture for a continuous composite at constant volume fractions of fibers. For that purpose, complete fiber length distributions are measured for each composition. The retained fiber lengths are typically between $0.02 \mathrm{~mm}$ to $0.8 \mathrm{~mm}$. Frequency of fibers are plotted as a function of fiber length and the number average fiber lengths are calculated [17]. The average retained fiber lengths for the composite formulations with each type of fiber reinforcements used $\left(1 / 4,1 / 8\right.$, and $1 / 16^{\prime \prime}$ chopped fibers) are given in Table 3 .

Verification of reinforcement efficiency can now be done by comparing the experimental values of well-defined lengths with those predicted by the rule of mixture for a continuous composite at constant volume fractions of fibers. A graph

Table 3. Average retained fiber length $(\mathrm{mm})$.

\begin{tabular}{|c|c|c|c|}
\hline \multirow[b]{2}{*}{ Matrix } & \multicolumn{3}{|c|}{ Type of Chopped Fiberglass Used } \\
\hline & $1 / 16^{\prime \prime}$ & $1 / 8^{\prime \prime}$ & $1 / 4^{\prime \prime}$ \\
\hline PS & 0.151 & 0.388 & 0.466 \\
\hline $25 \%$ PPO & 0.146 & 0.205 & 0.278 \\
\hline $50 \%$ PPO & 0.164 & 0.245 & 0.279 \\
\hline $65 \%$ PPO & 0.136 & 0.308 & 0.287 \\
\hline $75 \%$ PPO & 0.131 & 0.274 & 0.253 \\
\hline $85 \%$ PPO & 0.098 & 0.245 & 0.244 \\
\hline PPO & 0.124 & 0.238 & \\
\hline
\end{tabular}




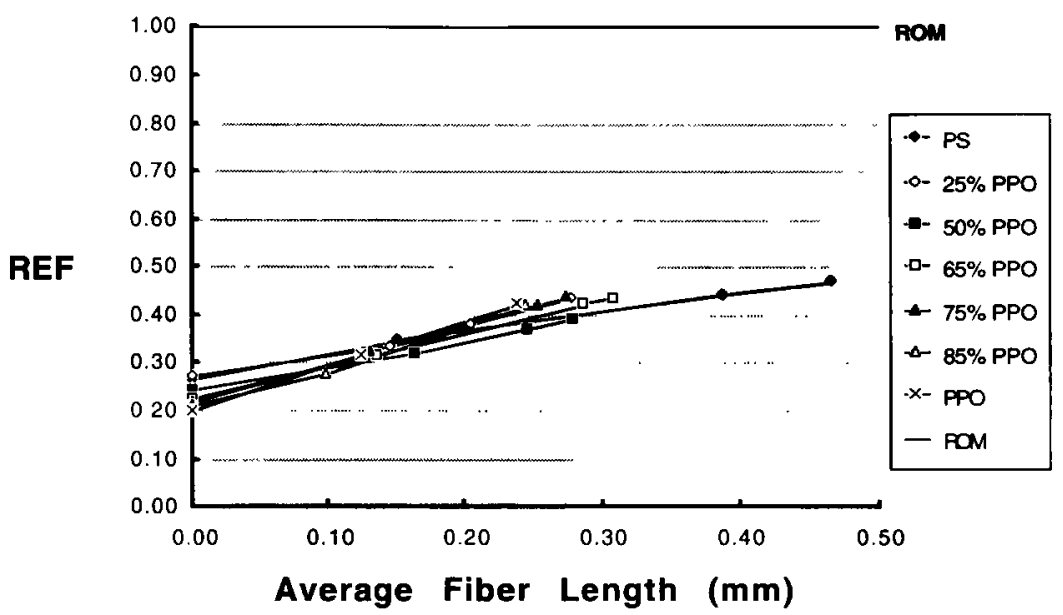

Figure 1. Reinforcement efficiency factor for stiffness as a function of fiber length.

is shown in Figure 1, similar to one given by Anderson and Lavengood [18] for an epoxy resin. The reinforcement efficiency factor (REF) is given as the ratio of the actual composite modulus to the rule of mixtures prediction and plotted as a function of average fiber length. This graph confirms that the elastic modulus is a strong function of fiber length for the range of fiber lengths considered. REF increases monotonically as the length of the retained fibers increases. As the fibers approach a length close to $0.5 \mathrm{~mm}, 50 \%$ reinforcement efficiency is reached. However, apparently longer fibers are needed to have a longitudinal modulus near the value for a continuous fiber composite at the same fiber loading (9.3 v/o).

It is a well established concept that the composite elastic modulus is dependent upon the fiber volume fraction. A similar dependence is also observed for the matrix ductility. To show the effects of matrix ductility on the composite elastic modulus, a composite elastic modulus is plotted against the composition at constant fiber volume fraction and fiber length in Figure 2. We observe an almost parallel stiffening effect over the brittle matrix composition range. However, stiffening becomes more effective as the ductility (or the PPO content) of the matrix increases. This points out a better stress transfer efficiency for the ductile matrix.

\subsection{Predictions of the Mechanics}

It was pointed out earlier that one of our aims is to verify the theories describing the influence of fiber length (or fiber aspect ratio) on the composite stiffness for SFRTP. So far, no work has been reported about the confirmation of the predictions of stiffness as a function of fiber length in well characterized SFRTP. By using different lengths of chopped fibers, we generated injection molded samples 


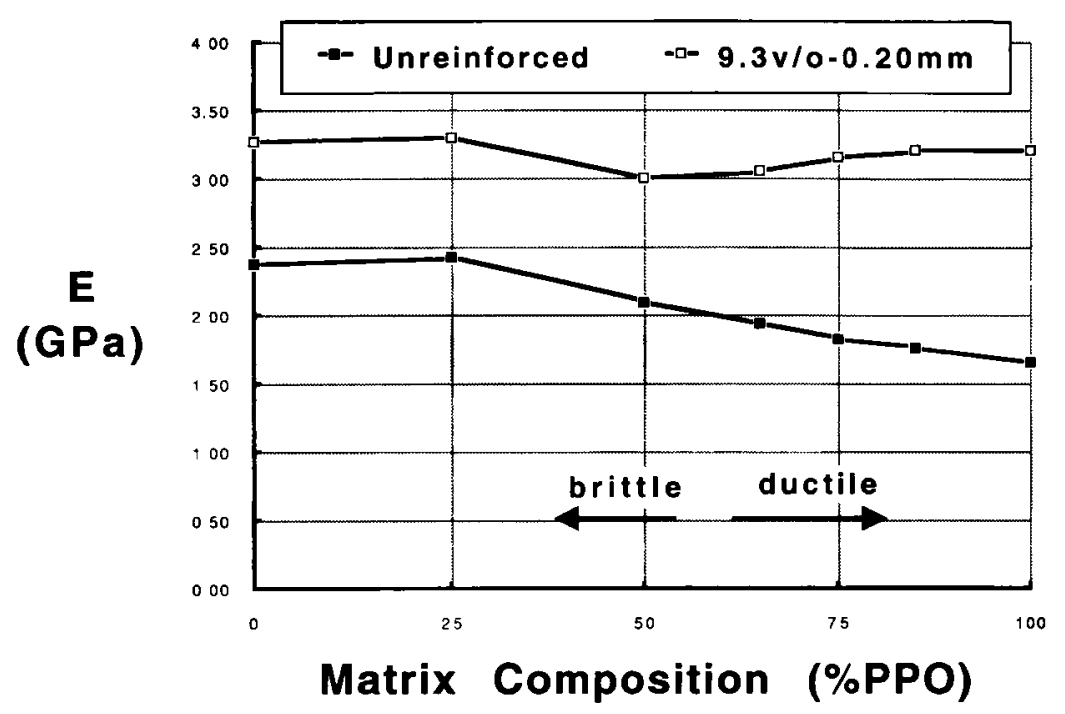

Figure 2. Effect of matrix ductility on the composite stiffness.

in a range of $0.0 \mathrm{~mm}$ to $0.5 \mathrm{~mm}$ average fiber lengths where composite stiffness varies significantly.

The stiffness predictions are compared with the experimental data for varying fiber lengths at constant fiber volume fraction. The three theoretical models, Equations (2), (6), and (8), which will be called Cox, H\&T and O\&W models, are used for the predictive calculations. Since the repetitive manipulation of theoretical models for varying fiber lengths is quite time-consuming, the calculations were eased with a computer. The calculated ROM value represents the upper limit of stiffness for a continuous, unidirectional fiber reinforced composite. Since these models are developed for the uniaxially aligned composites, they are compared first to the experimental data with the assumption that injection molded samples had perfect fiber alignment along the applied stress direction, i.e., before FOD is taken into account.

Experimental composite stiffness for PS is compared at constant fiber volume fraction $(9.3 \%)$ with the calculated values in Figure 3. The theoretical models predict higher than the experimental values. This is not surprising since these models are developed for uniaxially aligned composites and they do not take FOD into consideration. The following observations deserve special comment. The models predict a strictly nonlinear relation between stiffness and fiber length and they approach ROM value asymptotically. The O\&W model predicts the highest stiffness and Cox model predicts the lowest or closest to those of experimental values. However, at a certain fiber length there is a crossover between the Cox and H\&T models after which the H\&T model predicts the lowest stiffness. At this crossover point $(\ell=0.35 \mathrm{~mm})$, Cox and H\&T models predict $77 \%$ 
higher than the experimental value while the O\&W model predicts $100 \%$ higher value.

It should be pointed out here that experimentally measured fiber diameters are used in the equations for the theoretical calculations. A change in fiber diameter might be considered of little consequence in controlling composite stiffness. However, the aspect ratio (instead of the fiber length) is the controlling factor, and this is the most sensitive range for its effect on the composite stiffness. Obviously, it is not safe to use literature values of fiber diameter when examining the validity of composite theories. Therefore, this is yet another parameter which must be measured on the samples used in these experiments.

The distribution of fiber orientation was determined [17] following the procedure explained in Section 3. The complete FOD histograms for PS reinforced with three types of chopped fibers are presented in Figure 4. In this figure, $y$-axis is the flow direction and the fraction of fibers oriented at any direction is represented in $10^{\circ}$ intervals. For instance, if there was a random fiber orientation distribution, this histogram would be a circle representing equal fiber fractions at every direction. It is seen in this figure that the fraction of fibers oriented along the flow direction increases as the fiber length increases.

The FOD results show there is a fair degree of fiber alignment along the flow axis for the compositions, however, the alignment is not as perfect as has often been assumed for injection molded samples.

We will explore next whether misalignment is high enough to account for the difference between the experimental values and the stiffness predicted by the three models. Therefore, the models are used with the "Laminated Plate Theory" and abbreviated as Cox/LPT, H\&T/LPT, and O\&W/LPT. Krenchel's treatment of Cox model [Equation (9)] is also considered and abbreviated as Cox $/ \mathrm{Kr}$.

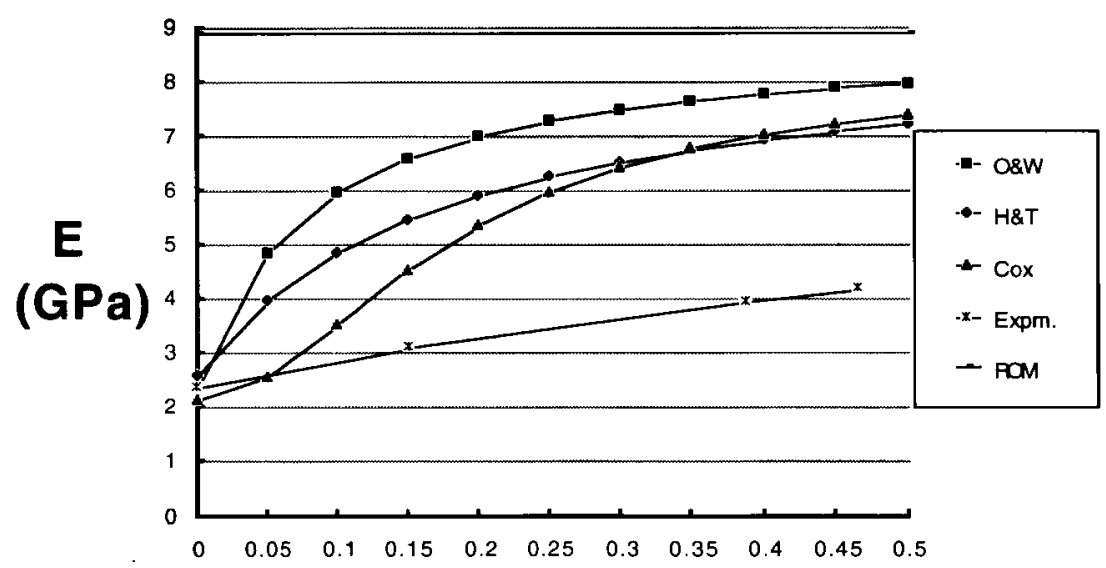

\section{Average Fiber Length $(\mathrm{mm})$}

Figure 3. Theoretical prediction of stiffness for PS as compared to experimental values at 9.3\% fiber volume fraction before FOD is taken into account. 


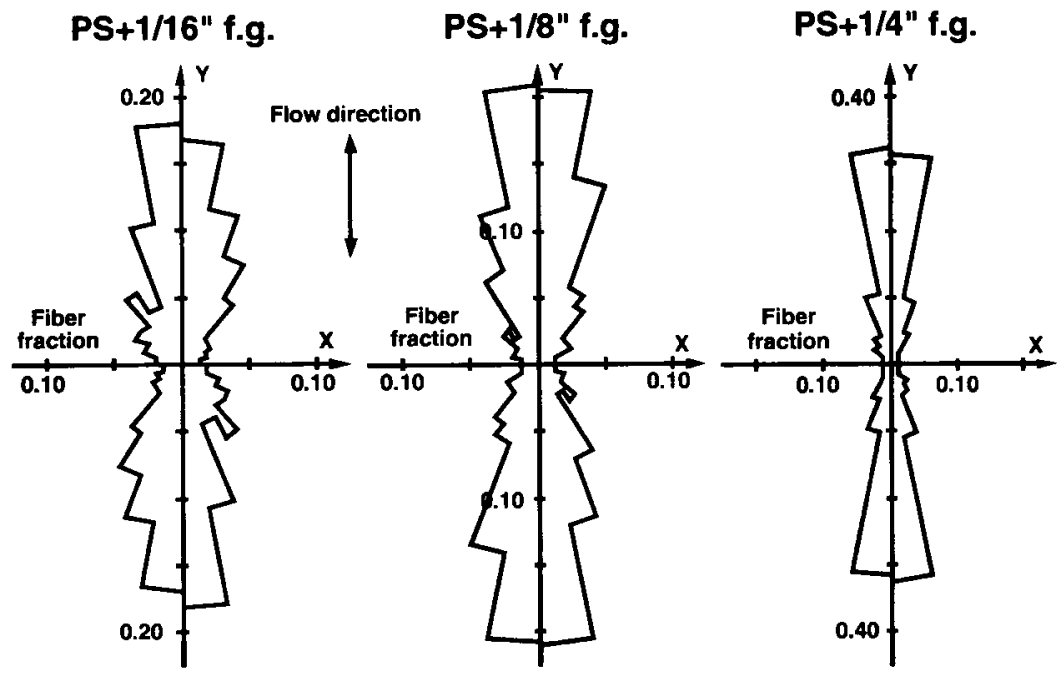

Figure 4. FOD histograms for PS.

Experimental composite stiffness for PS is compared with the predicted values after FOD is taken into account in Figure 5. It is readily apparent that the predicted values are much closer to the experimental values now. Cox/LPT model predicts the lowest or closest to the experimental values while O\&W/LPT model still predicts the highest stiffness. Although $\mathrm{Cox} / \mathrm{Kr}$ model begins predicting the

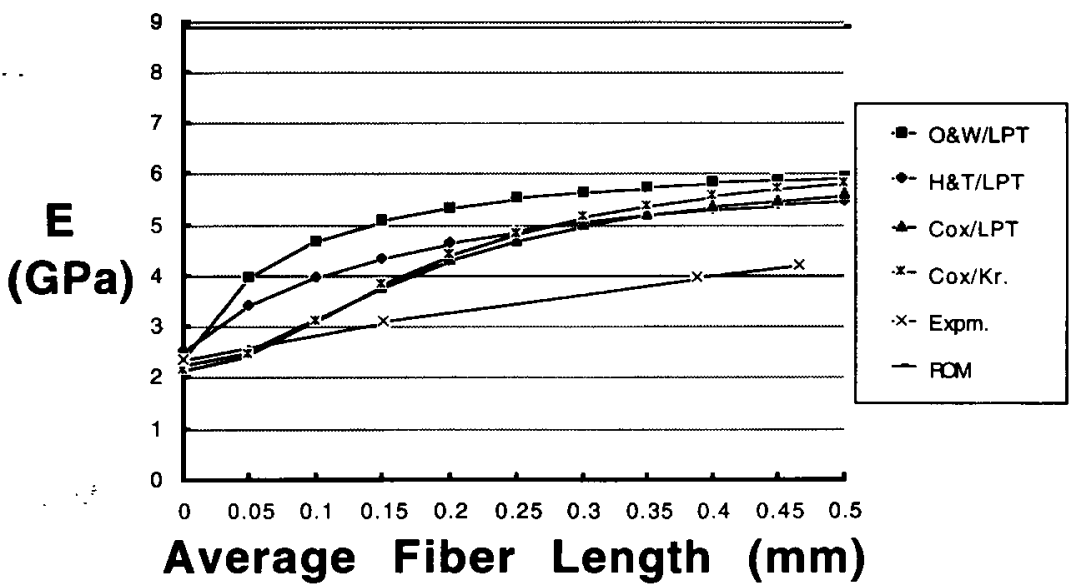

Figure 5. Theoretical prediction of stiffness for PS as compared to experimental values at 9.3\% fiber volume fraction after FOD is taken into account. 
lowest values with Cox/LPT model, it departs from Cox/LPT at $\ell=0.15 \mathrm{~mm}$ and predicts higher values. H\&T/LPT begins predicting higher values than Cox/ LPT and Cox $/ \mathrm{Kr}$, but catches up with Cox $/ \mathrm{LPT}$ at $\ell=0.35 \mathrm{~mm}$ and beyond which predicts the lowest values with Cox/LPT. At the same fiber length $(\ell=$ $0.35 \mathrm{~mm}$ ), Cox/LPT and H\&T/LPT now predict only $35 \%$ higher than the experimental value while Cox $/ \mathrm{Kr}$ and O\&W/LPT predict 42 and $51 \%$ higher. This shows that a big part of the difference between the predicted and experimental values can be accounted for by taking FOD effects into consideration. The improvement in the predictions can be attributed partly to the success of the laminate analogy, i.e., being able to treat the injection molded samples as an assembly of laminates.

The comparison of the predicted values to the experimental stiffness for the rest of the compositions after FOD is taken into account is given in Figure 6. Again, the predicted values are closer to the experimental values after FOD is taken into account. The order of closeness to the experimental values which each model predicts for PS does not change for the rest of the matrix compositions. However, closeness of the models to the experimental values changes for different compositions. There seems to be a better agreement between the predicted and experimental values as the ductility (or the PPO content) of the matrix increases. This may be attributed again to better stress transfer efficiency for the ductile matrix. The closest model, Cox/LPT, to the experimental value at $\ell=0.2 \mathrm{~mm}$ predicts $35 \%$ higher value for $25 \%$ PPO and 50\% PPO compositions (both are brittle matrix compositions). In the ductile matrix compositions, predictions are closer to the experimental values. As PPO content increases from $65 \%$ to $75 \%$ and $85 \%$, Cox/LPT model predicts only 25,15 , and $10 \%$ higher values than the experimental ones at $\ell=0.2 \mathrm{~mm}$. Both Cox $/ \mathrm{LPT}$ and $\mathrm{Cox} / \mathrm{Kr}$ models predict the stiffness perfectly for PPO.

In conclusion, we have shown that any realistic predictive work for injection molded SFRTP has to include not only the effects of fiber length distribution (FLD) but also the fiber orientation distribution (FOD). Although the theories predict generally higher values than those gotten experimentally, the agreement improves as the FOD is taken into account and as the matrix ductility increases. The results are more pleasing than if the calculated values were below the experimental values, in which case the presence of any voids or defects, etc., could not have been explained.

We have also shown there is a dependence between the composite stiffness and matrix ductility. The stiffening effect improves as the ductility of the matrix increases. REF is also a strong function of retained fiber length.

\section{ACKNOWLEDGEMENTS}

The work described was carried out in the Department of Materials Science and Engineering at the University of Michigan and we wish to acknowledge the help supplied by the technical staff of the Department. We are grateful to G.E. Plastics for supplying the additive free PPO. The helpful discussions with Professor Albert Yee are also gratefully acknowledged. 

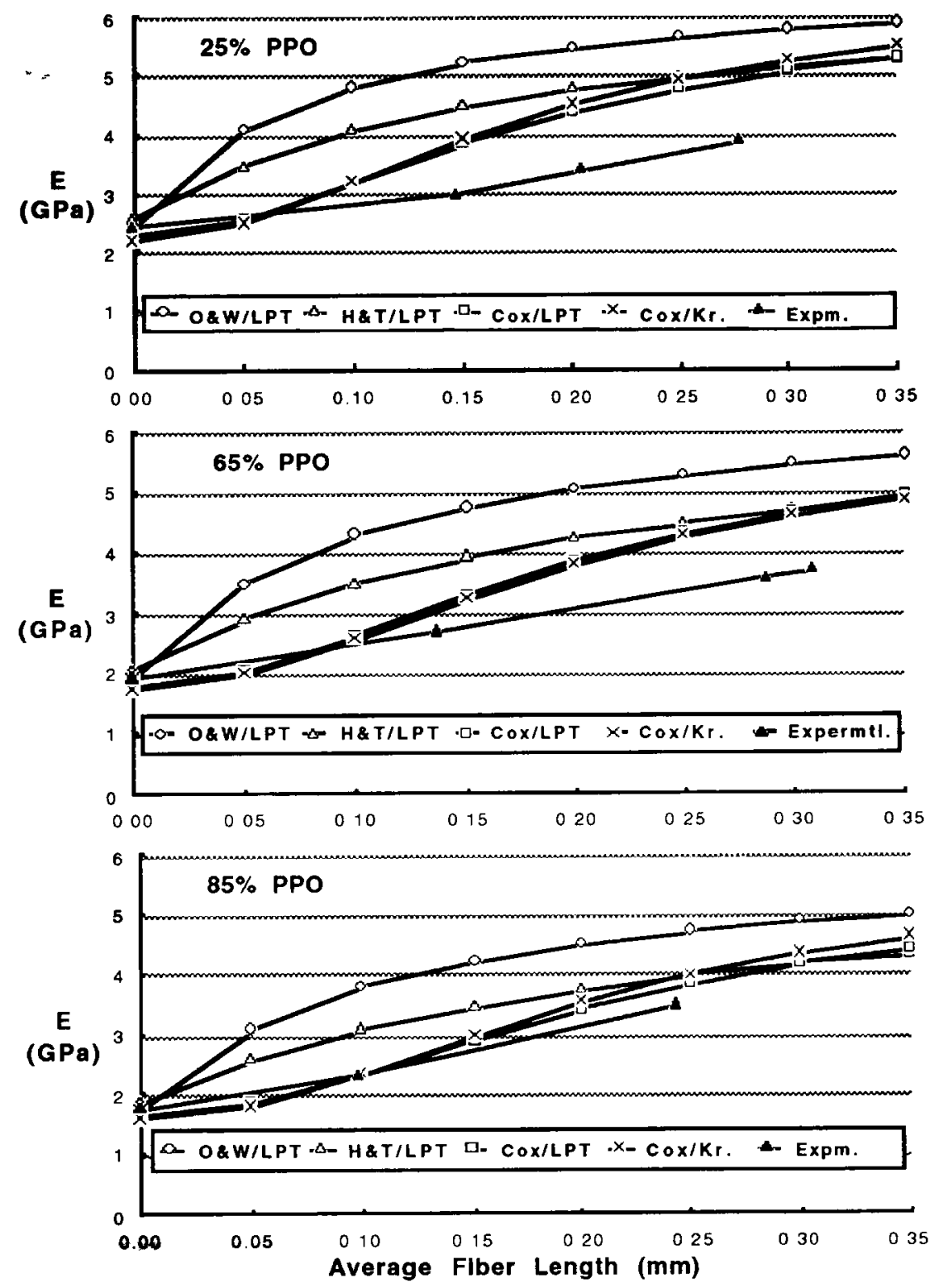

Figure 6. Theoretical prediction of stiffness for the rest of the compositions as compared to the experimental values at $9.3 \%$ fiber volume fraction after FOD is taken into account. 

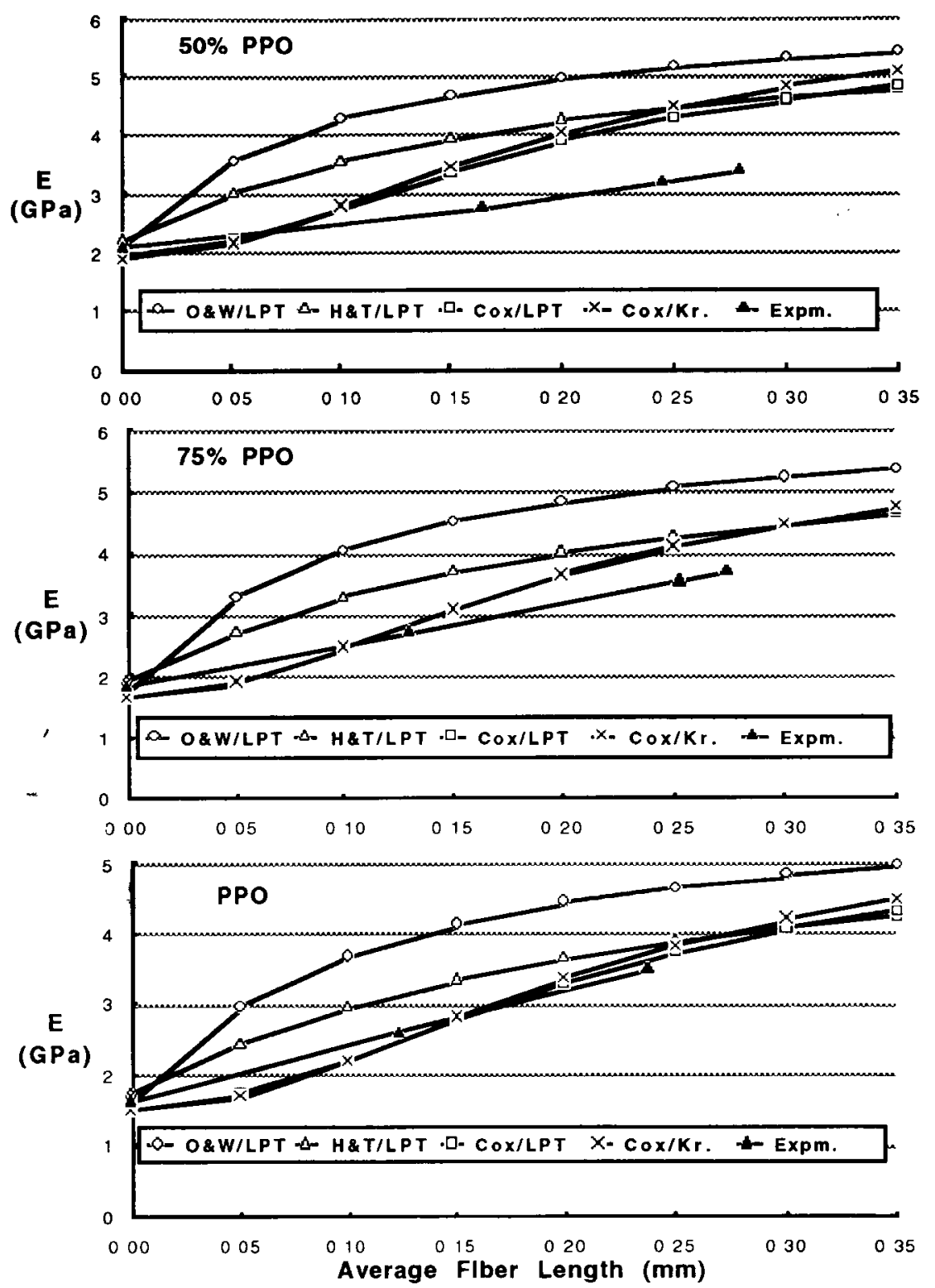

Figure 6 (continued). Theoretical prediction of stiffness for the rest of the compositions as compared to the experimental values at $9.3 \%$ fiber volume fraction after FOD is taken into account. 


\section{REFERENCES}

1. Prest, W. M. and R. S. Porter. 1972. J. Polym. Sci., A-2, 101:639.

2. Yee, A. F. 1977. Polym. Eng. Sci, 17:213.

3. Wellinghoff, S. T. and E. Baer. 1977. J. Polym. Sci. Phys. Ed., 15:1913.

4. Wellinghoff, S. T. and E. Baer. 1978. J. Appl. Polym. Sci., 22:2025.

5. Yee, A. F. and M. A. Maxwell. 1980. J. Macro. Sci., B17(3):543.

6. Serrano, A. M., G. E. Welsch and R. Gibala. 1982. Polym. Eng. Sci., 22:946.

7. Cox, H. L. 1952. Brit. J. Appl. Phys., 3:72.

8. Halpin, J. C. and J. L. Kardos. 1976. Poly. Eng. Sci., 16:344.

9. Hermans, J. 1967. Koninkl. Nederl. Akademic Proceedings, Series B, 70:1.

10. Hill, R. 1963. J. Mech. Phys. Solids, 11:357; ibid., 12:199, 1964.

11. Ogorkiewicz, R. M. and G. W. Weidmann. 1974. J. Mech. Eng. Sci., 16:10.

12. Counto, V. J. 1964. Mag. Concrete Res., 16:129.

13. Krenchel, H. 1964. Fiber Reinforcement. Copenhagen: Akademisk Forlag.

14. Halpin, J. C. and N. J. Pagano. 1969. J. Comp. Mat, 3:720.

15. Holik, A. S., R. P. Kambour, S. Y. Hobbs and D. G. Fink. 1979. Microstruct. Sci., 7:357.

16. Tekkanat, B. and R. Gibala. To be published.

17. Tekkanat, B. 1987. Ph.D. Dissertation, University of Michigan, Ann Arbor, MI.

18. Anderson, R. M. and R. E. Lavengood. 1968. S.P.E. Journal, 24:20. 